\title{
Dynamics and rheology of Janus drops in a steady shear flow
}

\author{
Misael Díaz-Maldonado and Ubaldo M. Córdova-Figueroa* \\ Department of Chemical Engineering, University of Puerto Rico-Mayagüez, Mayagüez PR 00681, USA
}

\begin{abstract}
The behavior and rheology of a dispersion of Janus drops (or Janus emulsion) under a steady shear flow are explored in the infinite dilution limit. To achieve analytical progress, the Janus drops are assumed to consist of a pair of fluids bounded to hemispherical domains of equal radii. At 'freely' suspended conditions the Janus drops undergo periodic orbits in a shear flow that are intermediate to that of a solid sphere and a disk that depend on the viscosities of the internal fluids. Non-Newtonian behavior is found for this system on account of the anisotropic hydrodynamics of the Janus drops. The viscosity of the Janus emulsion that corresponds to the minimum energy of dissipation is analogous to that derived by Taylor (1932) Proc. R. Soc. A 138, 41-48 for a dispersion of simple drops. It is also found that an external force can induce the Janus drops to adopt a preferential orientation in a shear flow. Interestingly, a neutrally buoyant Janus drop with a displaced center of gravity can migrate lateral to the undisturbed shear flow; it is inferred that this phenomenon can lead to spatial-dependent rheology in pressure-driven flows.
\end{abstract}

Keywords: low-Reynolds-number flows, Janus drop, Non-Newtonian rheology

\section{Introduction}

An understanding of the rheological properties of heterogeneous systems (e.g. colloids, emulsions, gels, foams) is of central relevance to emerging technologies, industrial and 5 engineering applications. A classic example is the implementation of high performance damping mechanisms for racing cars by taking advantage of the rapid increase of the viscous properties of magneto-rheological fluids upon application of a magnetic field (Olabi and Grunwald, 2007).

10 It is also well-known that a myriad of consumer products, which are typically made up by colloidal dispersions and emulsions (Larson, 1999), have been generated as a result ${ }_{40}$ of the numerous experimental and theoretical studies that have elucidated their rich rheological properties.

15 For compound multiphase drops it is surprising that rheological studies appear to be lacking from both experimental and theoretical grounds despite their diverse number ${ }_{45}$ of applications. The high selectivity to mass transport of compound multiphase drops make them attractive for the 20 micro-encapsulation of cells (Shintaku et al., 2007), drugdelivery and food processing applications (Vehring, 2008), purification of water resources $(\mathrm{Li}, 1971)$, micro-reactors ${ }_{50}$ (Bringer et al., 2004), and single-cell analysis (Joensson and Andersson, 2012) to mention a few examples. Compound multiphase drops have also found important applications in the fabrication of particles with precisely engineered features (Wang et al., 2011), materials process- ${ }_{55}$

\footnotetext{
* Corresponding author

Email address: ubaldom.cordova@upr.edu (Ubaldo M. Córdova-Figueroa)
}

ing (Morton et al., 1990; Lee et al., 2001; Tian et al., 2011), self-propulsion at the microscopic scale (Shklyaev, 2015), have served as a useful model to study the dynamics and rheological properties of blood cells (Kan et al., 1998; Hoskins et al., 2009), among several other applications of engineering interest (Johnson and Sadhal, 1985).

In practice, it is the rheology of a complex fluid that ultimately determines if it meets the requirements for its intended application. Despite of the diverse number of applications of compound multiphase drops mentioned above, it appears that there are few studies concerned on the rheology of this complex fluid. The first contributions on this subject have been conducted by Davis and Brenner (1981) and Stone and Leal (1990) who studied the rheology of double emulsions - also known as globules or encapsulated drops - in linear flows. Rheological studies of partly engulfed compound multiphase drops (snowman-like drops) seem to be missing; possibly because of the complexity of the drop shape in the calculation of the $3 \mathrm{D}$ flow field. Thus, the principal purpose of this study is to close the gap towards the determination of the rheology of partly engulfed multiphase drops in a steady shear flow via the analysis of a simpler, yet representative member of this class - the Janus drop. The term Janus, which takes after the mythological god of two faces present in ancient Roman literature, highlights that the drop consists of two parts of equal geometry but of different properties.

Microfluidic techniques are quite popular for the fabrication of Janus drops because of the precise control one can attain over the size of the drops such that it is a feasible task to produce a dispersion of Janus drops with 
a nearly uniform size distribution (Dendukuri and Doyle, 60 2009; Choi et al., 2012; Guzowski et al., 2012; Jeong et al., 2015). Recently, Zarzar et al. (2015) generated Janus drops with a diverse number of interesting features such as magnetic, light and $\mathrm{pH}$-sensitive properties with the aid ${ }_{120}$ of reconfigurable emulsification techniques and microflu-

65 idics. The collection of these recent accomplishments open venues of opportunities to the use of Janus drops in multidisciplinary research and to the development of emulsions with adaptive rheological properties. Therefore, the study ${ }_{125}$ of the rheology of Janus drops is not limited to the descrip-

70 tion of the rheological properties of compound multiphase drops; there is also an inherent motivation to do so because of the promising applications of Janus drops. The creation of Janus drops with specialized properties is a130 rapidly growing field, and therefore it is of interest to at-

75 tain an understanding on how these attributes can affect the rheology of Janus drops. This study aims to shed some light on how the fluid properties of Janus drops determine their dynamics and rheology in a steady shear flow.

In general, it is insightful to have some knowledge of the

80 flow at the microscopic level (individual particles or network structure) of complex fluids (colloidal dispersions, gels, emulsions, etc) to interpret the observed rheological behavior at the macroscopic scale. For compound multi-140 phase drops, there has been considerable progress on the

85 analytical treatment of axisymmetric flows in the limit of low-Reynolds-number flows (Johnson and Sadhal, 1985; Sadhal and Oguz, 1985; Morton et al., 1990; Chervenivanova and Zapryanov, 1989; Vuong and Sadhal, 1989;145 Kan et al., 1998; Tsemakh et al., 2004; Rosenfeld et al., 90 2009). Numerical studies have principally addressed the problem of simulating large changes in shape and breakup of compound multiphase drops subject to external flows in confinement and open domains (Bazhlekov et al., 1995;150 Smith et al., 2004; Zhou et al., 2008; Qu and Wang, 2012;

95 Hua et al., 2014). Typically changes in drop shape are considered as deviations from the initial equilibrium configuration in the absence of fluid motion. The theory to determine the drop shape at such static conditions was first ${ }_{155}$ introduced by Torza and Mason (1970), which is founded on the minimization of the interfacial energy of the compound multiphase drop. The analysis of the formation of compound multiphase drops in microfluidic settings has been performed by Zhou et al. (2006).

Particularly for Janus drops, Shardt et al. (2014) performed numerical simulations to study the dynamics of $2 \mathrm{D}$ Janus drops in a linear shear flow. Analytical progress on the determination of the hydrodynamics of Janus drops in an ambient flow has been achieved recently in a series165 of articles. In the first part of the series (Shklyaev et al.,

110 2013), the dynamics of a Janus drop in a uniform flow was analyzed as a function of the drop orientation and viscosity, where it is found that the Janus drop displays an anisotropic hydrodynamic resistance to the flow similar to that of a solid-spheroid of revolution. The second 115 part (Díaz-Maldonado and Córdova-Figueroa, 2015) ad- dresses the problem of a Janus drop subject to an ambient fluid that undergoes a linear shear flow. In that study the description of the hydrodynamics of the Janus drop is completed via the calculation of the grand resistance matrix developed by Brenner (1963). Thus, these two studies provide the essential information to analyze, from a theoretical point of view, the rheological properties (effective viscosity and differences in the normal stresses) of a Janus emulsion in the special limit the drops do not "see" each other. In other words, the macroscopic behavior of the Janus emulsion is determined by the dynamics of the individual drops.

In this investigation the rheology of Janus drops are probed under a steady shear flow and at infinite dilution conditions. The analysis is realized within the limit of no deflections to fluid-fluid interfaces because it is anticipated that such effects will only bring small departures (of the order of the capillary number) from Newtonian behavior, as in the case of single-phase drops (Schowalter et al., 1968). For this system, nonetheless, the anisotropic hydrodynamics of the Janus drops are found to produce significant non-Newtonian effects via differences in the normal stresses. Additionally, since the typical length scales of Janus drops in experiments are well above the sub-micron scale, stochastic effects associated to Brownian motion are entirely neglected in this study. The article is organized as follows. A model description is presented in Section 2. The preferential orientation of a Janus drop 'trapped' in a linear shear flow is studied in Section 3. The dynamics of a 'freely' suspended Janus drop is considered in Section 4, in which it is found that the Janus drop exhibits Jeffery-like orbits intermediate to that of a solid-sphere and a disk. The analysis of the cross-flow migration of a Janus drop in a linear shear flow is carried out in Section 5. From the results of this phenomenon it is inferred that a dispersion of multiphase drops in a Poiseuille flow (pressure-driven flow) can exhibit a non-uniform spatial distribution, and thus, spatially-dependent rheology. The article concludes in Section 6 with the calculation of the effective viscosity of a dilute Janus emulsion subject to a linear shear flow. Interestingly, it is found that the effective viscosity that corresponds to the minimum energy of viscous dissipation has an analogous form to that derived by Taylor (1932) for a dispersion single-phase drops. The difference is that the mean viscosity of the fluids that comprise the Janus drop replaces the drop viscosity in Taylor's result. In contrast to the rheology of double emulsions (Stone and Leal, 1990), non-Newtonian behavior is found in the non-deformable limit on account of the anisotropic hydrodynamics of Janus drops.

\section{Model description}

The simple model presented here for Janus drops is expected to be useful to attain some understanding on the dynamics and rheology of multiphase drops subjected to simple shear flows. A generalized schematic that depicts 


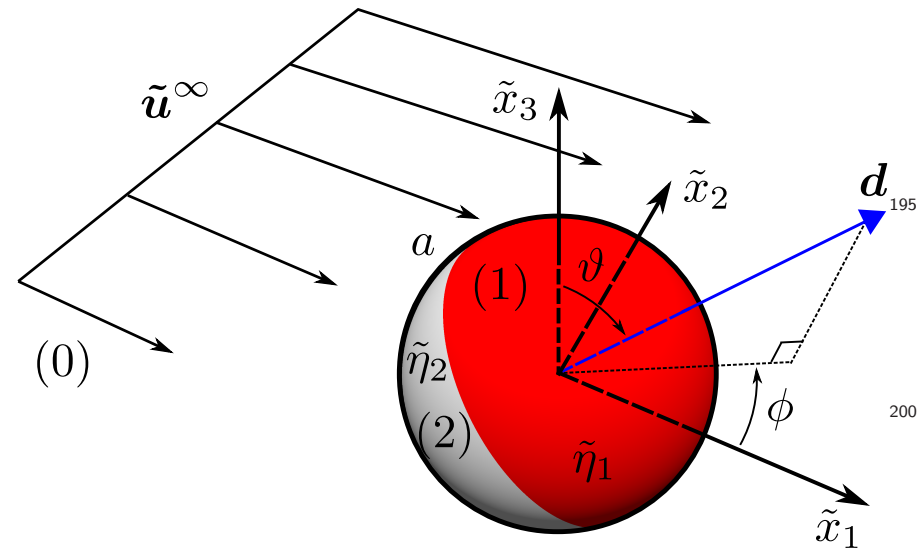

Figure 1: Unidirectional shear flow $\tilde{u}_{1}^{\infty}\left(\tilde{u}_{2}^{\infty}=\tilde{u}_{3}^{\infty}=0\right)$ past a 'per- ${ }_{205}$ fect' Janus drop of radius $a$. The fluids that constitute the Janus drop are confined to equally sized hemispheres. The symbol $(j)$ denotes the corresponding fluid phase of dynamic viscosity $\tilde{\eta}_{j}(j=0,1,2)$. The unit vector $\boldsymbol{d}$ denotes the direction of the axis of symmetry of the Janus drop, and it is defined with respect to a set of right-handed Cartesian axes fixed in space $\left(\tilde{x}_{1}, \tilde{x}_{2}, \tilde{x}_{3}\right)$. The components of this vector are related to the azimuthal $\vartheta$ and polar $\phi$ angles of a spherical ${ }^{210}$ coordinate system in the traditional manner.

the principal features shared in common by the problems considered in this study is provided in figure 1 . The sketch ${ }^{215}$ shows a 'perfect' Janus drop of radius $a$ immersed in a viscous ambient fluid ' 0 ' that executes a simple shear flow of non-zero mean velocity (uniform shear plus a streaming flow). The undisturbed velocity $\tilde{\boldsymbol{u}}^{\infty}$ is expressed in dimensional quantities and presented in index notation: ${ }^{220}$

$$
\tilde{u}_{1}^{\infty}=G \tilde{x}_{2}+U^{\infty}
$$

where $G$ is the applied shear rate, $U^{\infty}$ is the mean velocity of the undisturbed flow, and $\tilde{\boldsymbol{x}}$ is the position vec-225 tor. The notation to use a tilde on top of field variables (i.e. position, velocity, stress) and fluid properties (density and viscosity) is followed throughout this document to distinguish between their dimensional and non-dimensional form; constant quantities, such as the imposed shear rate eno $_{20}$ and streaming velocity, are shown without tilde for simplicity. The orientation of the Janus drop is given by the unit vector $\boldsymbol{d}$ (defined with respect to space-fixed axes) that is conventionally assumed to point towards the less viscous internal fluid $\left(\tilde{\eta}_{1} \leq \tilde{\eta}_{2}\right)$; this axis of symmetry is s235 conveniently used to characterize the drop orientation relative to the direction of the undisturbed flow.

The following assumptions pertain to this work: $(i)$ The pair of fluids ' 1 ' and ' 2 ' that comprise the Janus drop are bounded to equal hemispherical domains such that a flat 240 interface separates them. (ii) All fluids phases $(j=0,1,2)$ are immiscible, incompressible, and Newtonian (shear independent viscosity). (iii) The fluid-fluid interfaces are considered non-deformable and clean of surfactant agents.

(iv) Fluid motion occurs without the influence of inertia 245 effects. It suffices to point out that for the Janus drop to interface), the interfacial tension of the each internal fluid with the ambient has to be much larger than the interfacial tension with its counterpart (i.e. $\tilde{\gamma}_{10} \approx \tilde{\gamma}_{20} \gg \tilde{\gamma}_{12}$ ). (This constraint is not difficult to realize in practice; for example, Zarzar et al. (2015) utilized a three-phase system composed of hexane, perfluorohexane and water to produce nearly 'perfect' Janus drops because the interfacial tensions of hexane and perfluorohexane with water are similar.) Note that the conditions to have nearly 'perfect' Janus drops have been reported in great detail by others (Nisisako et al., 2006; Guzowski et al., 2012; Shklyaev et al., 2013) and therefore, it is not necessary to reproduce that information here. A complete theory for the determination of the shape and configuration of compound multiphase drops in the absence of fluid flows is provided by Torza and Mason (1970). For the remaining kinematical assumptions to hold valid, one only requires the capillary, $C a=\tilde{\eta}_{0} a G / \tilde{\gamma}_{12}$, and Reynolds number, $R e=\tilde{\rho}_{0} a^{2} G / \tilde{\eta}_{0}$, to be small, $\tilde{\eta}_{0}$ is the dynamic viscosity and $\tilde{\rho}_{0}$ is the density of the ambient fluid, and $\tilde{\gamma}_{12}$ is the interfacial tension of the internal fluids. This can be realized effortlessly in practice by application of a sufficiently small shear rate. Particularly, Torza and Mason (1970) carried out experimental observations of the dynamics of a Janus-like compound drop in a shear flow at reasonably small shear rates (around $0.1 \mathrm{~s}^{-1}$ ) to produce a low-Reynolds-number flow $\left(R e \sim 10^{-6}\right)$ constrained to a small capillary number in the order of magnitude of $10^{-1}$.

It is important to point out that the internal interface of the Janus drop is still subject to interfacial distortions because a flat surface cannot absorb the difference in the normal stress. Nevertheless, a simple scaling analysis is carried out here to demonstrate that a nearly flat interface can withstand the difference in the normal stress under the conditions of applicability of this study $(R e \ll 1$ and $C a \ll 1)$. A balance of the characteristic fluid stress $\tilde{\eta}_{0} G$ with the capillary pressure results in the sought expression for the radius of curvature $\tilde{R}$ of the internal interface: $\tilde{R} \sim \tilde{\gamma}_{12} / \tilde{\eta}_{0} G$. For example, for a Janus drop composed of hexane and perfluorohexane the value of interfacial tension at the internal interface $\left(\tilde{\gamma}_{12}=0.4 \times 10^{-3} \mathrm{~N} / \mathrm{m}\right)$ reported by Zarzar et al. (2015) is quite useful to provide a realistic estimate of the radius of curvature. For a Janus drop of radius $a=10^{-3} \mathrm{~m}$ suspended in water under a shear rate $G=10^{-1} \mathrm{~s}^{-1}$, the radius of curvature of the internal interface is found to be large compared to the drop size: $\tilde{R} / a \sim 10^{3}$; supporting the claim that a nearly flat interface can resist the difference in normal stress.

Even though the study of the changes in drop shape is of general interest, it is not included in this work because interfacial distortions are not expected to alter the qualitative behavior of the Janus drop in a linear shear flow. Only small quantitative departures, of the order of the capillary number, in the present results are anticipated. The analysis of the deflection of interfaces is more rewarding in bounded flows because of the expected coupling of the 
drop shape with its dynamical behavior.

The characteristic scales for length, fluid velocity and viscosity of the internal fluids is non-dimensionalized by the ambient viscosity $\eta_{1,2}=\tilde{\eta}_{1,2} / \tilde{\eta}_{0}$. Parameterization of the viscous properties of the Janus drop is conveniently realized via the mean drop viscosity $\eta=\left(\eta_{1}+\eta_{2}\right) / 2$ and viscosity contrast $\Delta \eta / 2 \eta=\left(\eta_{2}-\eta_{1}\right) /\left(\eta_{2}+\eta_{1}\right)$. It is clear that when the viscosity contrast is zero the Janus drop comprises fluids of equal viscosities $\left(\eta_{1}=\eta_{2}=\eta\right)$ such that the viscous properties are solely determined by the single parameter $\eta$. For non-zero $\Delta \eta / 2 \eta$ some interesting features of the Janus drop are found. One of such traits is considered in the next section, in which the conditions a Janus drop can adopt a preferential orientation in a simple shear flow are explored. An important application of this behavior is proposed in Section 6 to investigate the rheology of a dilute dispersion of Janus drops. Since the dynamics of the Janus drops must be known to study the rheology of this system, the analysis is incorporated in this work for completeness (Section 4).

\section{Preferential orientation of a Janus drop in a simple shear flow}

In this section the steady orientation of a Janus drop in a simple shear flow (deterministic behavior) is investigated. For the Janus drop to adopt a preferential orientation a hydrodynamic coupling must exist; to this end, an external force $\boldsymbol{F}^{e}$ must act at any location other than the hydrodynamic center of reaction $\boldsymbol{x}_{c r}$. For calculation purposes, it is assumed that the force passes through the geometrical center of the Janus drop. In general, this ensures that the center of reaction does not coincide with the geometrical center when the Janus drop is made up by fluids of different viscosities. It is also assumed that the force maintains the geometrical center of the Janus drop at a coincident arrangement to the centerline of the shear flow. The source of the external force is left unspecified since it is immaterial for the purposes of carrying out the analysis; however, this may be achieved in practice by means of gravity or optical traps. (There is a fair number of studies that have relied on optical trapping to maintain drops fixed in space in the presence of external flows; the interested reader is referred to Summers et al. (2006) and the references therein.) Note that the sole purpose of $\boldsymbol{F}^{\boldsymbol{e}}$ is to fix the location of the Janus drop in space; it only has an indirect influence over the preferential orientation adopted by the Janus drop in a simple shear flow. Depending on the shear to mean velocity $G a / U^{\infty}$, the mean drop viscosity $\eta$ and contrast $\Delta \eta / 2 \eta$, the Janus drop can exhibit a preferential orientation in a simple shear flow due to its lack of fore and aft symmetry (which is purely hydrodynamic in nature because the drop conforms to a spherical shape). The relevant point here is that the Janus drop experiences a torque from both the shearing and streaming motions of the ambient fluid that can balance each other at an appropriate shear to mean velocity ratio $G a / U^{\infty}$. Note that even in the limit of small values of viscosity contrast $(\Delta \eta / 2 \eta \ll 1)$, there is a shear to mean velocity ratio $G a / U^{\infty}$ that can be found such that the net torque on the Janus drop vanishes at some orientation $\boldsymbol{d}$. It is important to note that $\boldsymbol{d}$ must initially lie in the plane of the shear gradient of the undisturbed flow $x_{1}-x_{2}$ plane (or equivalently $\vartheta=\pi / 2$ ) for this to occur; otherwise, there are additional torques along the $x_{1}$ and $x_{2}$-axes that cannot be balanced by the streaming velocity for evident reasons such that a preferential orientation cannot occur. A simple approach to overcome this arrangement issue is to fabricate a neutrally buoyant Janus drop with an eccentric center of gravity; the Janus drop will tend to position $\boldsymbol{d}$ in the plane of shear if gravity is also in the plane of shear. (However, one would need to account for the gravitational torque on the final orientation of the Janus drop.) An advantage of this setup is that the drop orientation can be characterized exclusively by the polar angle $\phi$.

The analysis presented here entirely neglects the dynamics of rotation of the Janus drop prior to reaching its steady orientation, since it is obvious that the preferential orientation is independent of such initial conditions, as in the case of spheroidal particles in an extensional flow, which consistently become aligned with one of the principal axes regardless of the initial positioning (Leal, 1980). This allows for a simple analysis to the problem by seeking an orientation $\boldsymbol{d}$ that is torque-free. The procedure to determine the torque and force on the Janus drop follows the classic direct approach of integrating the fluid stress at the outer surface of the drop. The constitutive equation of a Newtonian fluid is used to calculate the stress from the velocity field of the ambient fluid, which is coupled to the motion of the internal fluid phases of the Janus drop via the so-called Stokes equations. Variation of the undisturbed flow with the drop orientation $\boldsymbol{d}$ is taken into account by projecting the laboratory frame-based flow into a set of axes affixed to the Janus drop via a transformation matrix. Without further preliminary remarks, the Stokes equations are presented (in non-dimensional form) for each fluid phase with respect to a spherical coordinate system $(r, \vartheta, \phi)$ that takes advantage of the geometry of the Janus drop:

$$
\begin{array}{r}
\boldsymbol{\nabla} \cdot \boldsymbol{u}^{(j)}=0, \quad-\nabla p^{(j)}+\eta_{j} \nabla^{2} \boldsymbol{u}^{(j)}=0, \\
\boldsymbol{n} \cdot \boldsymbol{u}^{(j)}=0,[\boldsymbol{u}]=[\boldsymbol{n} \cdot \boldsymbol{\sigma}]=\mathbf{0}, \text { at } r=1, \\
\boldsymbol{n} \cdot \boldsymbol{u}^{(1,2)}=0,[\boldsymbol{u}]=[\boldsymbol{n} \cdot \boldsymbol{\sigma}]=\mathbf{0}, \text { at } \vartheta=\pi / 2, \\
\boldsymbol{u}^{(0)}=\boldsymbol{u}_{\infty} \text { as } r \rightarrow \infty,
\end{array}
$$

where $\boldsymbol{n}$ is the unit normal vector at fluid-fluid interfaces; at the outer drop surface it is directed outward, and at the internal interface it points from fluid ' 2 ' to fluid ' 1 '. The quantities $\boldsymbol{u}^{(j)}$ and $p^{(j)}$ designate the respective velocity and pressure fields of the $j$ th fluid phase. The stress tensor is that of a Newtonian fluid (in index notation): $\sigma_{l k}^{(j)}=$ 
$-p^{(j)} \delta_{l k}+\eta_{j}\left(\nabla_{l} u_{k}^{(j)}+\nabla_{k} u_{l}^{(j)}\right)$, where $\delta_{l k}$ is the identity tensor. At interfaces the normal component of the fluid velocity is zero $\left(u_{n}=0\right)$ because of the immiscibility of the fluids (no mixing of the fluid phases). The brackets ${ }^{310}$ designate a jump of the enclosed quantity at interfaces. At the outer surface $(r=1)$ the jump is directed from the internal to the ambient fluid, and at the internal interface $(\vartheta=\pi / 2)$ it is directed from the inner fluid ' 2 ' to fluid ' 1 '. At distances far from the Janus drop $(r \rightarrow \infty)$ the flow ${ }^{315}$ reduces to the undisturbed velocity $\boldsymbol{u}_{\infty}$.

Integration of the surface tractions at the outer drop surface $(r=1)$ yields the hydrodynamic force $\boldsymbol{F}$ on the Janus drop:

$$
\boldsymbol{F}=\oint_{S_{d}} \boldsymbol{n} \cdot \boldsymbol{\sigma} \mathrm{d} S_{d}
$$

where $S_{d}$ is the area of the outer drop surface. The torque $\boldsymbol{T}$ is given by the first moment of the surface tractions:

$$
\boldsymbol{T}=\oint_{S_{d}} \boldsymbol{x} \times(\boldsymbol{n} \cdot \boldsymbol{\sigma}) \mathrm{d} S_{d} .
$$

Prior to the presentation of results, it is instructive to consider the preferential orientation of the Janus drop $\mathrm{in}_{330}$ the absence of the shearing motion of the ambient fluid.

275 In such a situation the imposed flow reduces to a uniform flow along the direction of the $x_{1}$-axis: $\tilde{\boldsymbol{u}}^{\infty}=U^{\infty} \boldsymbol{e}_{1}$. It is important to recall that there is an external force $\boldsymbol{F}^{\boldsymbol{e}}$ that prevents the Janus drop from undergoing trans- ${ }_{335}$ lational motion, and that there are no restrictions on its

280 rotation. For the purposes of providing physical arguments it is sufficient to bring to the discussion the main findings of the behavior of the Janus drop with different internal viscosities in a uniform streaming flow; the technicalities of this problem has been reported by Shklyaev et al. (2013).

${ }_{285}$ Now consider the symmetry axis of the Janus drop $\boldsymbol{d}$ to lie in the $x_{1}-x_{2}$ plane at an arbitrary direction with respect to the undisturbed flow given by the polar angle $\phi$. From the perspective of an observer the Janus drop ro- ${ }_{345}$ tates (due to the coupling of $U^{\infty}$ with the torque) until

$290 \boldsymbol{d}$ becomes aligned with the $x_{1}$-axis. This turns out to be a configuration where the flow is axisymmetric everywhere, and consequently, torque-free regardless of the internal viscosities of the Janus drop. Note that this is true ${ }_{350}$ for any body of revolution with broken fore and aft symmetry; classic examples are the spherical cap (Dorrepaal, 1978) and the aggregate of unequally sized spheres (Nir and Acrivos, 1973). Therefore, it is expected that the axis of symmetry of the Janus drop will be nearly aligned with the $x_{1}$-axis in the limiting case of small shear to mean velocity ratio $\left(G a / U^{\infty} \ll 1\right)$. It is important to point out that these arguments only apply to a Janus drop of unequal internal viscosities; in the ideal situation of $\eta_{1}=\eta_{2}$, the Janus drop behaves as a symmetric spheroid of revo- ${ }_{360}$ lution with no tendency to rotate in a streaming flow $\boldsymbol{U}^{\infty^{3}}$ such that there is no preferential orientation. It follows from this observation that there must be a hydrodynamic coupling of the torque $\boldsymbol{T}$ with the streaming velocity $\boldsymbol{U}^{\infty}$ for the Janus drop to adopt a steady orientation in a simple shear flow.

Figure $2 a$ shows the preferential orientation (via the polar angle $\phi$ ) with increasing $G a / U^{\infty}$ of a Janus drop of mean viscosity $\eta=1$ and constant values of viscosity contrast $\Delta \eta / 2 \eta$. For small ratios of the shear to mean velocity $\left(G a / U^{\infty} \ll 1\right)$ the angle $\phi$ grows linearly with $G a / U^{\infty}$, in agreement with the above arguments for vanishing shearing rates. It is seen that the Janus drop tends to align its axis of symmetry in the direction of the velocity gradient of the undisturbed flow $\left(x_{2}\right.$-axis $)$ for all values of the viscosity contrast $\Delta \eta / 2 \eta$ as $G a / U^{\infty}$ is increased. In this arrangement the torque imparted by the streaming flow (which opposes that of the shear) reaches a maximum value. This becomes apparent by noting that the uniform flow can be broken down into transversal and axisymmetric components about $\boldsymbol{d}$, and that only the transversal component opposes the torque exerted by the shearing motion of the ambient fluid. Beyond this point, further increase of $G a / U^{\infty}$ results in a situation where the torque exerted by the shear is greater than that of the uniform flow, such that a preferential orientation cannot occur; the Janus drop simply rotates indefinitely with a periodic speed.

The dependence of the preferential orientation of the Janus drop with $G a / U^{\infty}$ and the mean drop viscosity $\eta$ at a fixed viscosity contrast $\Delta \eta / 2 \eta=0.4$ is presented in figure $2 b$. It is readily seen that there is a non-monotonic dependence of the preferential orientation with the mean drop viscosity. Interestingly, a Janus drop of relatively low mean viscosity $(\eta=0.1)$ reaches a transversal arrangement of $\boldsymbol{d}$ relative to the direction of the undisturbed flow $(\phi=\pi / 2)$ at higher shearing rates $G a / U^{\infty}$ in comparison with more viscous drops. An explanation to this behavior is that the shear flow becomes less effective on generating a torque than the streaming flow in the low viscosity limit. For Janus drops of greater viscosity the torque exerted by the shearing motion of the ambient is substantial in comparison to that imparted by the streaming counterpart, and therefore, the shear to mean velocity $G a / U^{\infty}$ required to arrange the Janus drop into a particular orientation is less.

It is important to point out that without some trapping mechanism it may prove to be difficult to maintain fixed the position of the Janus drop in space. Note that in general the net hydrodynamic force on the Janus drop is not necessarily parallel to the flow because of the anisotropic resistance of the drop (Shklyaev et al., 2013). Nonetheless, if the axis of symmetry of the Janus drop $\boldsymbol{d}$ is aligned with the $x_{2}$-axis the net hydrodynamic force on the Janus drop acts parallel to the direction of the flow $\left(x_{1}\right.$-axis), and therefore, a simple body force (such as gravity) may be used to balance the hydrodynamic forces on the Janus drop. The results that follow correspond to this particular situation.

Figure $3 a$ illustrates the dependence of $G a / U^{\infty}$ on $\Delta \eta / 2 \eta$ for constant values of the mean drop viscosity $\eta$. 

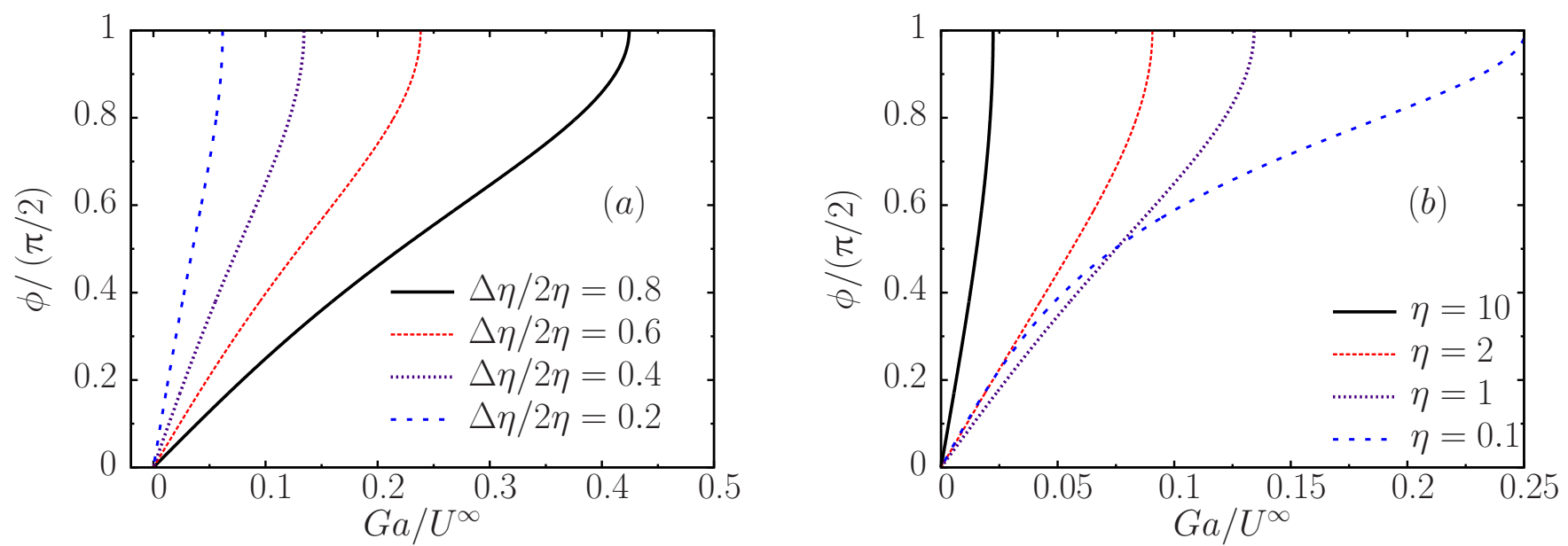

Figure 2: (Colors online) Influence of the shear to mean velocity ratio $G a / U^{\infty}$ on the preferential orientation of a Janus drop of (a) constant mean viscosity $\eta=1$ with selected values of $\Delta \eta / 2 \eta$, and $(b)$ constant $\Delta \eta / 2 \eta=0.4$ with selected values of $\eta$.
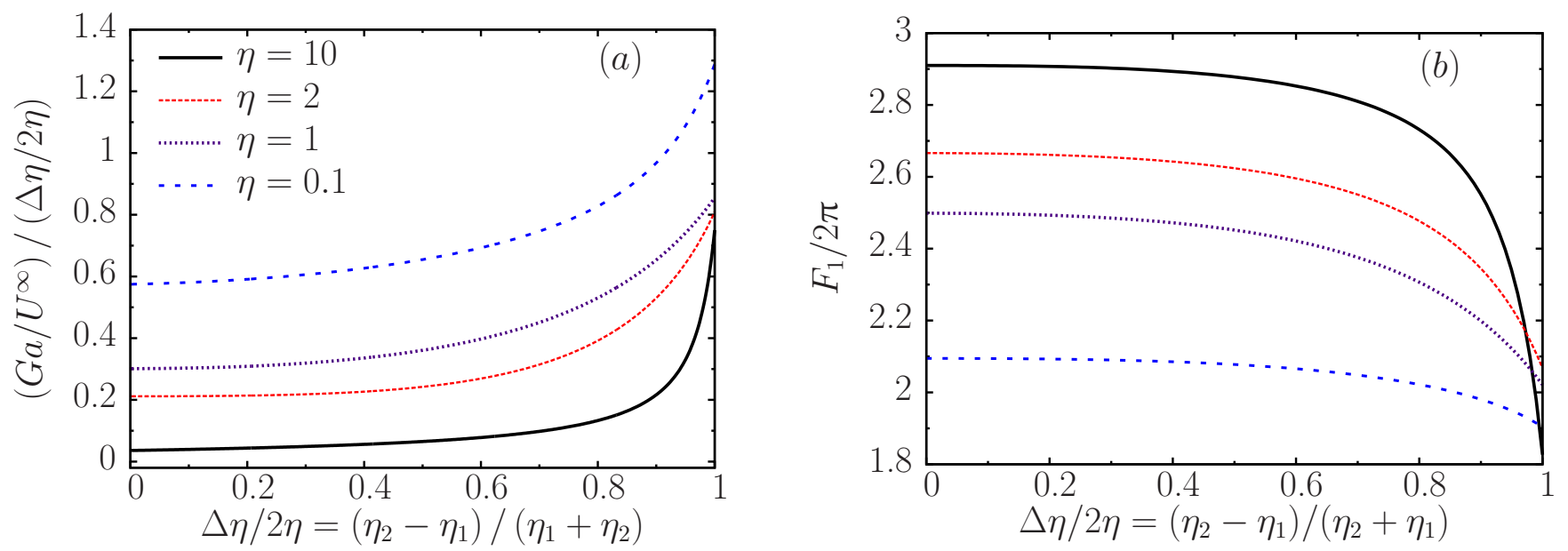

Figure 3: (Colors online) The effect of increasing viscosity contrast $\Delta \eta / 2 \eta$ at fixed values of the mean drop viscosity $\eta$ on ( $a$ ) the shear to mean velocity $G a / U^{\infty}$ and $(b)$ the net hydrodynamic force $F_{1}$ (in units of $\tilde{\eta}_{0} a U^{\infty}$ ).

In order to improve the presentation of results $G a / U^{\infty}$ is

scaled with $\Delta \eta / 2 \eta$. Note that the flat curvature for small

$\Delta \eta / 2 \eta$ suggests a linear dependence of the shear to mean 3 velocity $G a / U^{\infty}$ with the viscosity contrast. The apparent monotonic dependence of $G a / U^{\infty}$ with the mean drop viscosity is a consequence of the shear-torque decreasing more rapidly with $\eta$ than the torque imposed by the uniform flow.

The influence of $\Delta \eta / 2 \eta$ on the net hydrodynamic force $F_{1}$ (in units of $\tilde{\eta}_{0} a U^{\infty}$ ) is shown in figure $3 b$ for selected values of the mean drop viscosity $\eta$. It is seen that the net force is nearly independent of the viscosity contrast in the limit of small viscosity contrast $(\Delta \eta / 2 \eta \ll 1)$; in fact, the 395 force on the Janus drop reduces to that on a single-phase drop $\tilde{F}_{1}=2 \pi(2+3 \eta) /(1+\eta) \tilde{\eta}_{0} a U^{\infty}$. This can be understood from a unification of the results of Shklyaev et al. (2013) and Díaz-Maldonado and Córdova-Figueroa (2015); basically, the force imparted by the shearing motion of the ambient is $O(\Delta \eta / 2 \eta)$; whereas, the force contribution by the streaming motion of the ambient flow predominates because it is $O(1)$. A marked decrease on the force is not seen until both $\Delta \eta / 2 \eta \rightarrow 1$ and $\eta \gg 1$; however, its application may be limited to exceptional situations in practice, as in Jeong et al. (2015) because one of the internal phases is significantly more viscous than its counterpart. Note that in general the viscosities of the internal fluid phases are quite similar (for instance see Nisisako et al. (2006); Zarzar et al. (2015)); therefore, it is anticipated that most of the behavior of Janus drops in experiments can be understood from the theory at small viscosity contrast $(\Delta \eta / 2 \eta \ll 1)$. The predicted reduction of the net hydrodynamic force arises because the shear-force acts opposite to the direction of the imposed shear flow. 


\section{Dynamics of a 'freely' suspended Janus drop in a linear shear flow}

The dynamics of a 'freely' suspended Janus drop in a linear shear flow of a Newtonian fluid is investigated with respect to the fixed reference frame $\left(x_{1}, x_{2}, x_{3}\right)$. The main steps to arrive at the equations that govern the dynamics of the Janus drop are presented here since these will prove to be useful when studying the cross-streaming velocity of the Janus drop in the next section. On account of the linearity property of Stokes flow, the dynamics of a Janus drop in a general ambient flow are determined by an exact balance of the net hydrodynamic force and torque on a stationary Janus drop in the imposed flow with those on a Janus drop undergoing solid-body motions in a quiescent fluid. It is advantageous to choose the free center of rotation $\boldsymbol{x}_{c r}$ as a reference to carry out the present analysis since it turns out that drop translation and rotation are decoupled due to the zero coupling tensor at that location (Díaz-Maldonado and Córdova-Figueroa, 2015). Therefore, it follows that the dynamics of the Janus drop in a linear shear flow is analogous to that of a symmetric spheroid of revolution with an apparent aspect ratio $r_{h}$ that depends on the internal viscosities of the Janus drop. The equation that describes the rotational dynamics of the Janus drop can be readily derived from the resultant torque $\boldsymbol{T}$ on the Janus drop (presented in non-dimensional form and in index notation):

$$
\begin{aligned}
T_{k} & =\left[X^{C} d_{k} d_{l}+Y^{C}\left(\delta_{k l}-d_{k} d_{l}\right)\right]\left(\Omega_{l}^{\infty}-\omega_{l}\right) \\
& -Y^{H} \epsilon_{k l n} d_{n} d_{m} E_{m l}^{\infty}=0,
\end{aligned}
$$

where $\Omega_{l}^{\infty}$ is the rotational velocity of the ambient fluid, $\omega_{l}$ is the rotational velocity of the Janus drop, $E_{m l}^{\infty}$ is the undisturbed rate-of-strain tensor, $\epsilon_{k l n}$ is the permutation tensor, $X^{C}$ is the resistance of the Janus drop to undergo rotation about its axis of symmetry, $Y^{C}$ is the resistance of the Janus drop to rotation transversal to its axis of symmetry, and $Y^{H}$ defines the shear-torque imposed by the rate-of-strain field. Notably, $X^{C}$ is inherently zero in contrast to other bodies of revolution because the Janus drop behaves as a single-phase drop in this case (DíazMaldonado and Córdova-Figueroa, 2015). The values of the resistance functions can be found in the cited article. The transcendental equation for the orientation dynamics of the Janus drop can be derived by operating the cross product on both sides of the above equation with the unit vector $d_{j}$, followed by the use of the well-known identity $\epsilon_{k l n} \epsilon_{k j i}=\left(\delta_{l j} \delta_{n i}-\delta_{l i} \delta_{n j}\right)$ (Deen, 1998) to arrive at the sought expression, which is identical to that of a spheroidal ${ }^{400}$ particle (Kim and Karrila, 2005):

$$
\dot{d}_{i}=\omega_{l} d_{j} \epsilon_{l j i}=\Omega_{l}^{\infty} d_{j} \epsilon_{l j i}+B\left[E_{i m}^{\infty} d_{m}-E_{j m}^{\infty} d_{m} d_{j} d_{i}\right],
$$

where here the dot symbol on top of a variable denotes its differentiation with respect to time, and $B=Y^{H} / Y^{C}$ is commonly referred to as the Bretherton constant of the equivalent spheroid (Bretherton, 1962). It is important to point out that this result is in accordance with the experimental observations of Torza and Mason (1970) of a freely rotating Janus-like compound drop in a linear shear flow. The time evolution of the axis of symmetry $\boldsymbol{d}$ of the partly engulfed drop closely follows the rotation dynamics of a 'freely' suspended spheroid of revolution. An interesting aspect of their observations relevant to this work is that despite the difference in viscosities of the compound drop it behaves as a symmetric spheroid of revolution, which supports the notion that compound drops rotate about the free center of rotation under 'freely' suspended conditions. A final remark that deserves attention is that the experiments demonstrated that interfacial deflections only brought small quantitative deviations to the dynamics of the compound drop in comparison to spheroids of revolution; this finding justifies the assumption of treating interfaces as non-deformable for the purposes of analyzing the dynamics of compound drops in the limit of small capillary number.

Following Kim and Karrila (2005), one can express the components of $\boldsymbol{d}$ with respect to the azimuthal $\vartheta$ and polar $\phi$ angles of the spherical coordinate system (the angles are related to the set of space-fixed axes $\left(x_{1}, x_{2}, x_{3}\right)$ in the usual manner); then, after some elementary operations one obtains the following system of differential equations that characterize the orientation vector $\boldsymbol{d}$ in space-time (shown in non-dimensional form):

$$
\begin{aligned}
& \dot{\vartheta}=\frac{1}{4}\left(\frac{r_{h}^{2}-1}{r_{h}^{2}+1}\right) \sin 2 \vartheta \sin 2 \phi, \\
& \dot{\phi}=-\frac{1}{r_{h}^{2}+1}\left(r_{h}^{2} \sin ^{2} \phi+\cos ^{2} \phi\right) .
\end{aligned}
$$

For convenience the Bretherton constant has been expressed with respect to the apparent hydrodynamic aspect ratio $r_{h}$ via the archetypal relation $B=$ $\left(r_{h}^{2}-1\right) /\left(r_{h}^{2}+1\right)$. The solution to the system of differential equations was first presented by Jeffery (1922) for a spheroidal particle with its axis of revolution initially placed in the $x_{1}-x_{3}$ plane $(\phi(t=0)=0)$ :

$$
\begin{aligned}
\tan \vartheta & =\frac{C}{\left(r_{h}^{2} \sin ^{2} \phi+\cos ^{2} \phi\right)^{\frac{1}{2}}}, \\
\tan \phi & =r_{h}^{-1} \tan \left(\frac{G t}{r_{h}+r_{h}^{-1}}\right),
\end{aligned}
$$

which describes closed orbits in space-time of period $\tilde{\tau}=$ $2 \pi G^{-1}\left(r_{h}+r_{h}^{-1}\right)$. The constant $C$ is known as the orbital constant (Kim and Karrila, 2005) that parametrizes the initial orientation $\boldsymbol{d}$ along the azimuthal axis. When $C=0$ the Janus drop traces an equatorial orbit in an imaginary unit sphere, and as $C \rightarrow \infty$ the Janus drop orientation approaches a degenerate orbit with $\boldsymbol{d}$ aligned with the vorticity axis of the undisturbed shear flow $\left(x_{3}-\right.$ axis). The periodic orbits of a Janus drop of equal internal 

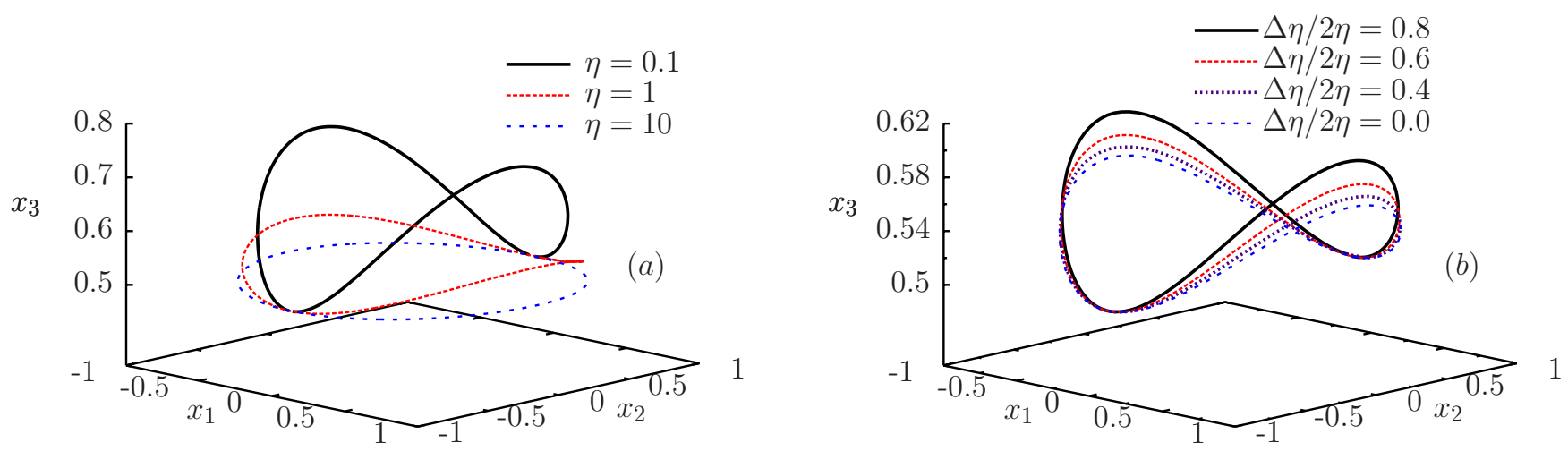

Figure 4: (Colors online) (a) Dependence of period orbits with the mean drop viscosity of a Janus drop with equal internal viscosities $\eta_{1}=\eta_{2}$. (b) The effect of the viscosity contrast $\Delta \eta / 2 \eta$ on the periodic orbits for a constant mean drop viscosity $\eta=1$.

viscosities $\left(\eta_{1}=\eta_{2}=\eta\right)$ are shown in figure $4 a$ for an initial drop orientation $\vartheta(t=0)=\pi / 3$, and $\phi(t=0)=\pi / 2$. As one may expect, the Janus drop undergoes changes in orientation in a linear shear flow of an effective spheroidal (or top) particle (Jeffery, 1922; Saffman, 1956). It is readily seen that as the mean drop viscosity $\eta$ increases, the periodic orbits of the Janus drop approach those of a solid sphere (in which the axis of revolution $\boldsymbol{d}$ describes a cone about the $x_{3}$-axis). In this limit the torque imposed by the straining flow vanishes to zero such that the drop orientation is determined by the angular motion of the ambient fluid (i.e. $\dot{\boldsymbol{d}}=\boldsymbol{\Omega}^{\infty} \times \boldsymbol{d}$ and $r_{h}=1$ as $\eta \rightarrow \infty$ ). The influence of the viscosity contrast on the periodic orbits of a Janus drop with a constant mean viscosity of $\eta=1$ is presented in figure $4 b$. (The same initial conditions are used to generate the curves.) The observed shift (or increase in eccentricity) of the periodic orbits as $\boldsymbol{d}$ swepts out of the plane of shear $\left(x_{1}-x_{2}\right.$ plane) with increasing values of $\Delta \eta / 2 \eta$ arises because of the reduction of the torque due to the rotational motion of the ambient fluid in comparison to its straining counterpart. This shift represents a change in the dynamical behavior of the Janus drop from a nearly ${ }^{445}$ isotropic body (sphere-like) to a slender oblate spheroid (disk-like); particularly, $r_{h}=0.75$ for $\Delta \eta / 2 \eta=0.8$. Note that the degree of the shift depends greatly on the viscous properties of the Janus drop, such that it is expected to be less pronounced for more viscous Janus drops of relatively similar internal viscosities. The changes in the dynamical behavior of the Janus drop are best characterized by the eccentricity factor $\varepsilon=\sqrt{1-r_{h}^{-2}}$, which is zero for a sphere $(\varepsilon=0)$ and tends to unity $(\varepsilon \rightarrow 1)$ for a disk-like spheroid. A plot of the eccentricity factor for a Janus drop with constant values of the mean viscosity $\eta$ and increasing viscosity contrast is presented in figure 5 . From the figure, it follows that the dynamical behavior of a Janus drop lies between the extremes of a solid sphere and a disk.

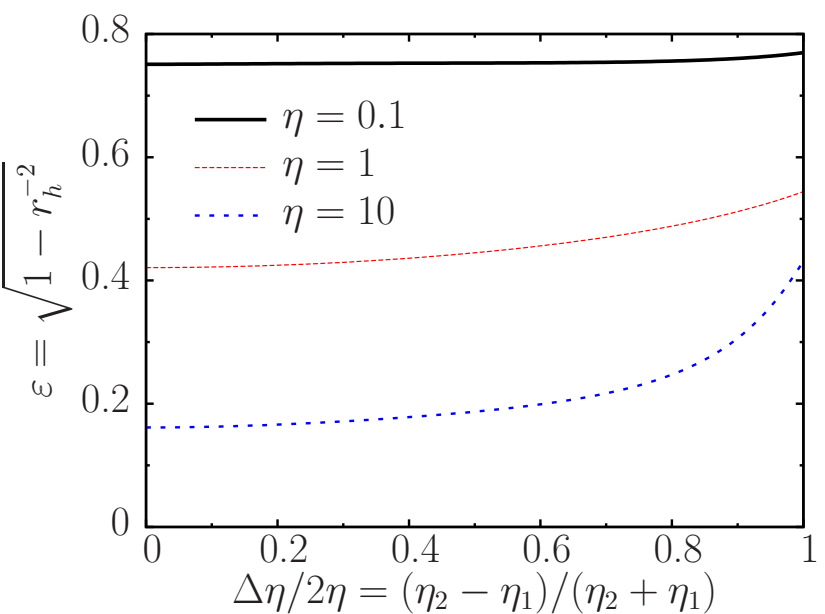

Figure 5: (Colors online) Apparent eccentricity factor as a function of the viscosity contrast $\Delta \eta / 2 \eta$ for constant values of the mean drop viscosity $\eta$.

\section{Cross-streaming velocity of a Janus drop in a shear flow}

The objective of this section is to study the crossstreaming velocity of a Janus drop in a shear flow induced by the action of an external force $\boldsymbol{F}_{e}$ parallel to the streamlines of the undisturbed flow. In principle, it is the anisotropic resistance of the Janus drop to the streaming flow $\left(\boldsymbol{U}^{\infty}-\boldsymbol{v}\right)$ that furnishes the cross-streaming phenomena, where $\boldsymbol{v}$ denotes here the translational velocity of the Janus drop. A closely related situation is that of a spheroidal particle that sediments in a quiescent, viscous Newtonian fluid (Happel and Brenner, 1965). The analysis presented here follows an analogous treatment to that used in Section 4. The translational and rotational motions of the Janus drop are derived from an overall balance of the force and torque. It is assumed that the external force acts in such a manner that does not impose a torque on 
the Janus drop that permits Equation (6) to describe the 495 rotational dynamics. The time evolution of the position $\boldsymbol{x}$ of the Janus drop with respect to space-fixed coordinates reads:

$\dot{x}_{k}=\boldsymbol{v}_{k}=u_{k}^{\infty}+\left[\left(X^{A}\right)^{-1} d_{k} d_{l}+\left(Y^{A}\right)^{-1}\left(\delta_{k l}-d_{k} d_{l}\right)\right] F_{l, 500}^{e}$

where $X^{A}$ and $Y^{A}$ are the scalar functions that describe the resistance of the Janus drop to streaming flows parallel and perpendicular to the axis of revolution $\boldsymbol{d}$, respectively (Díaz-Maldonado and Córdova-Figueroa, 2015). Al-505 though not shown explicitly, the undisturbed velocity is evaluated at the center of free rotation $\boldsymbol{x}_{c r}$. Note that the external force $\boldsymbol{F}^{e}$ exclusively acts in the positive direction of the $x_{1}$-axis, and that the velocity components $v_{2,3}$ correspond to the cross-streaming velocity of the Janus drop. 510

455 (Naturally, $v_{1}$ is the velocity of the Janus drop along the streamlines of the undisturbed flow.) Perhaps it is even more relevant to note that the cross-streaming velocity is intrinsically coupled to both the orientation and position of the Janus drop because there is a consequential stream-515 460 ing velocity that arises from the displacement of the drop from the centerline of the shear flow $\left(x_{1}\right.$-axis). As a result, the complexity of this problem limits the analytical progress, which leaves one to rely on a numerical technique to obtain a solution. The results presented in this section520 are obtained by solving the system of differential equations with a fourth-order Runge-Kutta method. Other methods of comparable accuracy were used to validate the results; this is not shown here for the sake of brevity.

By bearing in mind that the results may be of some use 25 to practitioners, the cross-streaming velocity is reported when $\boldsymbol{d}$ lies in the plane of shear of the undisturbed flow $\left(x_{1}-x_{2}\right.$ plane). The justification for choosing this particular configuration is that this corresponds to one of the simplest experimental setups that can be devised to in-530 vestigate the cross-streaming velocity of a Janus drop for two principal reasons: $(i)$ the axis of revolution $\boldsymbol{d}$ stays in the plane of shear such that its angular motion can be measured with more ease, and ( $i i)$ there is only one component of the cross-streaming velocity $\left(v_{3}=0\right)$. Without incurring in a loss of generality, the mean velocity of the shear flow is set to zero $\left(U^{\infty}=0\right)$ such that the ambient flow corresponds to a pure shearing flow. It is assumed that at initial conditions $(t=0)$ the free center of rotation of the Janus drop is located at the origin of the set of space-fixed axes $\left(x_{1}=0, x_{2}=0, x_{3}=0\right)$ with $\boldsymbol{d}$ aligned with the $x_{2}$-axis. For calculation purposes only, the external force is defined to be equivalent to the drag force on a solid sphere of radius $a$ subject to a uniform flow of velocity $G a: \tilde{F}_{1}^{e}=6 \pi \tilde{\eta}_{0} G a^{2}$. Note that the qualitative bethe magnitude of the force.

The orientation of the axis of revolution $\boldsymbol{d}$ is plotted alongside the ratio of the cross-streaming velocity to the external force $\left(v_{2} / F_{1}^{e}\right)$ in figure $6 a$ as a function of the dimensionless time for a Janus drop with equal internal viscosities $\eta_{1}=\eta_{2}=\eta=1$. The plots show that the crossstreaming velocity is periodic in time and that it reaches peak values when the axis of revolution is at a diagonal with respect to the principal direction of the undisturbed shear flow $(\cos \phi= \pm \sqrt{2} / 2)$, and that it vanishes when it is aligned with any of the principal axes $x_{1}$ and $x_{2}$ due to the evident symmetry arguments. It can be deduced that at low shear rates, the limit of a Janus drop undergoing cross-flow migration in a streaming flow is recovered. For larger shearing rates, on the other hand, the crossstreaming velocity is quite small. This effect has interesting implications on a dispersion of Janus drops subjected to pressure-driven flows, in which it is inferred from the results of this study that the dispersion exhibits a nonuniform spatial distribution with a higher concentration of Janus drops near the duct boundary. In turn, this effect can lead to rheological properties that depend on the spatial distribution of the Janus drops. It is fitting to point out that spatially-dependent rheological properties of dispersions has been identified to be a problem of central importance of materials processing applications in industry (Chan and Leal, 1979).

The non-monotonic dependence of the cross-streaming velocity $v_{2}$ with the mean drop viscosity is evidently shown in figure $6 b$, where $v_{2}$ decays to zero as $\eta \rightarrow \infty$, as it should, since this corresponds to the limit of a solid sphere that has an isotropic resistance to the external flow. One interesting feature of the results is that the cross-streaming velocity consistently reaches peak values, regardless of the mean drop viscosity $\eta$, when the axis of revolution is at a diagonal with the direction of the undisturbed flow. The effect of the viscosity contrast $\Delta \eta / 2 \eta$ on the cross-streaming velocity has been found to be quite small under the range of viscous properties that Janus drops have in practice, and for this reason the results have been omitted.

\section{Constitutive equation of a dilute Janus emulsion in a steady shear flow}

The results of this section are expected to be useful to practitioners interested in studying the rheology of a dilute dispersion of Janus drops. In view of the various experimental studies (Torza and Mason, 1970; Nisisako et al., 2006; Choi et al., 2012; Guzowski et al., 2012; Zarzar et al., 2015) that have fabricated Janus drops with radii above the micron-size $(\gtrsim 10 \mu \mathrm{m})$, the stochastic effects associated to thermal fluctuations of the suspending fluid (Brownian motion) are entirely neglected in this study. (It is well known that particles above one micron in size are unaffected by Brownian motion (Russel et al., 1989).) For the purposes of gaining leading and relevant rheological information, it is appropriate to analyze the problem under the simplest scenario: a dilute dispersion of Janus drops subject to a steady shear flow of a Newtonian fluid without the effects of inertia and Brownian motion.

For a dispersion of bodies of revolution, as in this case, 

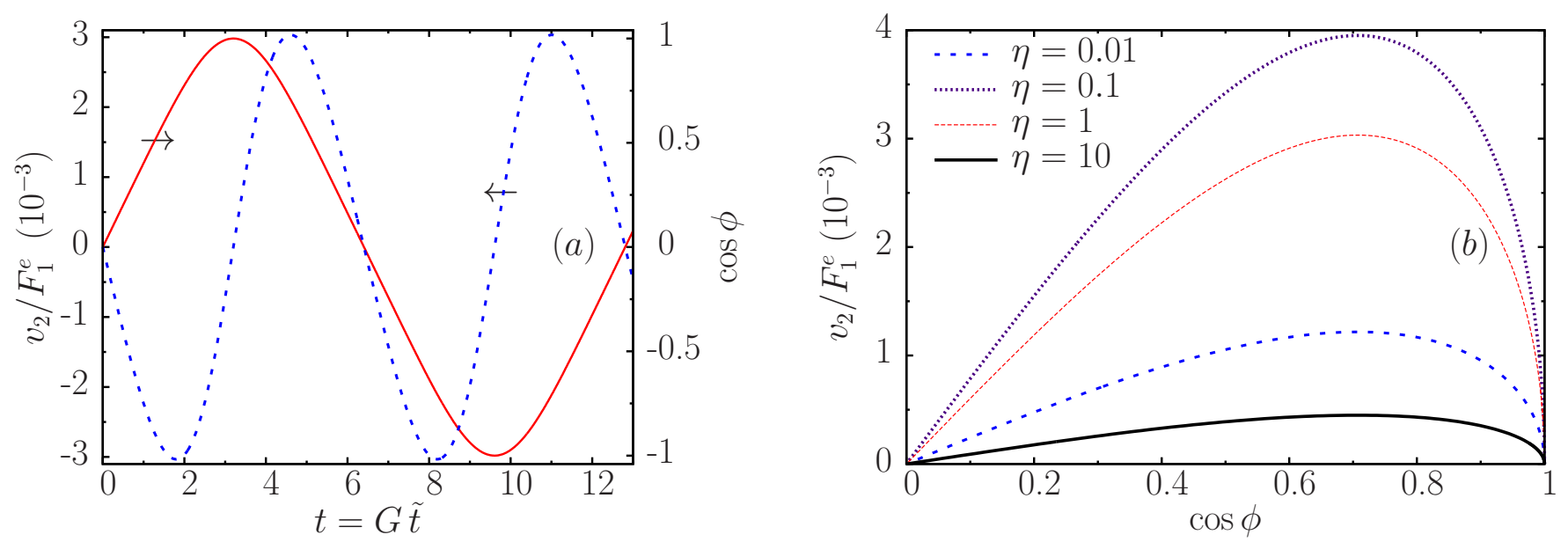

Figure 6: (Colors online) (a) The solid-line (red) depicts the dynamics of orientation and the dashed-line (blue) shows the non-dimensional ratio of the cross-streaming velocity with the external force. (b) Cross-streaming velocity as a function of the arrangement of the axis of revolution of the Janus drop for constant values of $\eta$.

the orientation distribution of the particles in suspension intrinsically determines the rheological properties observed at the macroscopic scale. This is the case because it is the disturbance-flow caused by the particles that lead to an apparent increase in the viscosity of the host fluid. As one may suspect, for certain orientations the particles produce greater disturbances to the flow than others such that it follows, from this simple argument, that the effective rheological properties of the dispersion must depend on the orientation distribution of the particles. The effect of the orientation distribution on the rheology of dispersions comprising bodies of revolution has been the subject of active research right after the publication of Jeffery (1922). For dispersions of sub-micron particles it is the relative importance of Brownian motion to the applied flow that determines the rheology of the dispersion, regardless of the the initial orientation distribution of the particles (Hinch and Leal, 1973; Brenner, 1974). On the other hand, in the complete absence of Brownian motion, particle-particle interactions and inertia effects, as in the present study, the orientation distribution is solely determined by the initial orientation of the particles via the so-called Jeffery-orbits. This is in fact, a consequence of the inability of the shear flow to set bodies of revolution under 'freely' suspended conditions into a preferential orientation at zero Reynolds number, as first noted by Jeffery (1922). One can take advantage of this instrinsic behavior of the flow to study the rheology under conditions in which the initial orientation distribution can be controlled. The most straightforward situation to realize this in practice is to have a dispersion with a uniform orientation distribution.

In principle, there are simple strategies that can be used to obtain a dispersion of Janus drops with a uniform orientation distribution; two are proposed here motivated by the latest achievements on the synthesis of Janus drops with designed properties (Zarzar et al., 2015). The use of neutrally buoyant Janus drops with an eccentric gravita- tional center is a simple method to obtain a dispersion with a uniform orientation distribution. To fabricate a neutrally buoyant drop with a center of gravity displaced from the geometrical center the internal fluids must be of differing densities but with a mean density $\tilde{\rho}$ that matches the density of the ambient fluid (i.e. $\tilde{\rho}=\left(\tilde{\rho}_{1}+\tilde{\rho}_{2}\right) / 2=\tilde{\rho}_{0}$ and $\left.\tilde{\rho}_{1} \neq \tilde{\rho}_{2}\right)$. The second method is to apply a magnetic field to a dispersion of magneto-responsive Janus drops. Both approaches offer the possibility of studying the rheology of Janus drops with fixed orientations in time; however, the corresponding analysis lies outside the scope of this study. From this point onward the constitutive equation for a dilute Janus emulsion is derived analytically under the mentioned conditions.

Following Batchelor (1970), the calculation of the bulk stress $\left\langle\Sigma_{i j}\right\rangle$ of a dispersion of 'freely' suspended particles in a linear shear flow involves a volume average of the contributions to the stress of the continuous fluid phase and the dispersed particles. Here, the term 'particles' has a general scope that encompasses both fluid and solid bodies. The constitutive equation for the bulk stress of a dispersion of particles in a fluid has an analogous form to that of an ordinary Newtonian fluid but with an additional contribution that arises from the presence of the particles (shown in non-dimensional form):

$$
\left\langle\Sigma_{i j}\right\rangle=-\langle p\rangle \delta_{i j}+2 \eta_{0}\left\langle E_{i j}\right\rangle+\left\langle S_{i j}^{p}\right\rangle
$$

where the angle brackets \langle\rangle denote a volume average of the enclosed quantity, and $\left\langle S_{i j}^{p}\right\rangle$ represents the particle contribution to the bulk stress and it is implicit that it involves a sum over all the particles in the dispersion. For a single particle, $S_{i j}^{p}$ is the hydrodynamic dipole plus an additional contribution that arises from the distribution of the rate-of-strain field at the surface of the particle (Kim and 
Karrila, 2005):

$$
S_{i j}^{p}=\oint_{S_{p}} n_{k} \sigma_{k i} x_{j} \mathrm{~d} S_{p}-\eta_{0} \oint_{S_{p}}\left(n_{i} u_{j}+n_{j} u_{i}\right) \mathrm{d} S_{p}
$$

where $n_{i}$ is the unit normal vector directed outward at each point over the surface of the particle, and $S_{p}$ is the outer surface of the particle. For force and torque-free particles in suspension the hydrodynamic dipole reduces to the stresslet: a symmetric tensor that captures the particle shape and long-range disturbances to the flow (Deen, 1998; Kim and Karrila, 2005). Note that the latter surface integral vanishes identically for a solid particle because the fluid velocity consists solely of solid-body motions at the surface of the particle. Although the superficial velocity of a fluid drop in a linear shear flow develops from the local rate-of-strain in addition to solid-body motions, the surface integral also vanishes on account of the symmetry of the rate-of-strain field. For Janus drops of identical internal viscosities $\left(\eta_{1}=\eta_{2}\right)$ the surface integral vanishes as well, based on the same symmetry arguments used for a single-phase drop. This finding holds well, even so, for Janus drops of different viscosities provided that $\eta$ adopts finite to moderate values, which is generally the case in practical situations. Therefore, the effect of the superficial distribution of the rate-of-strain field can be safely neglected. For a single Janus drop, the stresslet at an arbitrary drop orientation is given by the following expression:

$$
\begin{aligned}
& \frac{\tilde{S}_{i j}^{p}}{\frac{2}{3} \pi \tilde{\eta}_{0} G a^{3}}=c_{0} E_{i j}^{\infty}+c_{1}\left[\left(d_{i} E_{j k}^{\infty}+d_{j} E_{i k}^{\infty}\right) d_{k}\right. \\
& \left.-2 d_{i} d_{j} d_{k} d_{l} E_{l k}^{\infty}\right]+c_{2}\left(\epsilon_{i k l} d_{j}+\epsilon_{j k l} d_{i}\right) d_{l}\left(\Omega_{k}^{\infty}-\omega_{k}\right)
\end{aligned}
$$

where the above equation guarantees the stresslet to be a symmetric and traceless tensor, as it should be. The first term at the right-hand side of the equation corresponds to the stresslet of a simple fluid drop in a linear shear flow determined by Taylor (1932): $\tilde{S}_{i j}^{p}=2 \pi / 3 c_{0} \tilde{\eta}_{0} a^{3} \tilde{E}_{i j}^{\infty}=$ $2 \pi / 3 \tilde{\eta}_{0} a^{3}(4+10 \eta) /(1+\eta) \tilde{E}_{i j}^{\infty}$. However, in the context of this problem the drop has an equivalent viscosity $\eta=\left(\eta_{1}+\eta_{2}\right) / 2$. The additional terms are periodic contributions to the stresslet that depend on the orientation of the Janus drop $\boldsymbol{d}$ relative to the direction of the undisturbed shear flow. Plots of $c_{0}, c_{1}$ and $c_{2}$ for a Janus drop that satisfies $\eta_{1}=\eta_{2}=\eta$ are provided in figure 7 . It is notable that the stresslet functions $c_{1,2}=0$ as $\eta \rightarrow \infty$ such that the periodicity of the stresslet vanishes identically. Therefore, one can expect right away that the viscosity of a dispersion of hard spheres is recovered in this limit, in analogy with dispersions of single-phase drops (Taylor, 1932; Derkach, 2009). Note that the applicability of Equation (14) is expected to hold beyond the limit of equal internal viscosities on account of the weak dependence of the stresslet functions with the viscosity contrast $\Delta \eta / 2 \eta$ in the range $\eta \lesssim 10$. Figure 8 presents plots (that support this claim) of the stresslet functions against $\Delta \eta / 2 \eta$

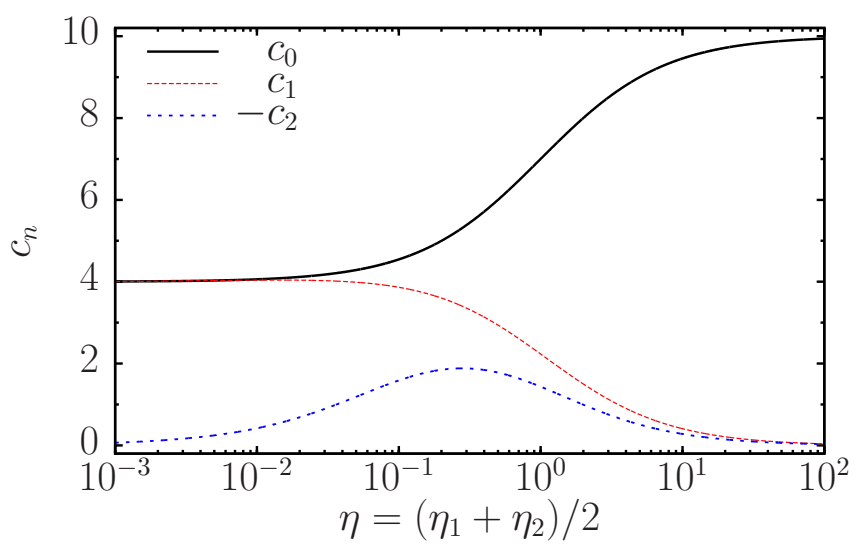

Figure 7: (Colors online) Variation of the stresslet functions $c_{n}$ $(n=0,1,2)$ with the mean drop viscosity $\eta$ for a Janus drop with equal internal viscosities.

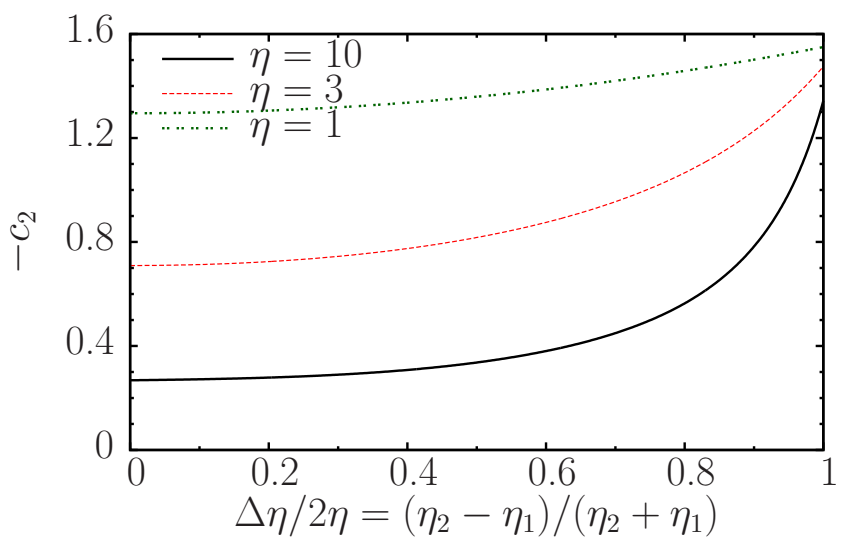

Figure 9: (Colors online) The dependence of the stresslet associated to drop rotation with the viscosity contrast and mean drop viscosity.

while keeping constant the mean drop viscosity $\eta$. In the low mean drop viscosity limit the stresslet functions retain nearly constant values regardless of the increase in $\Delta \eta / 2 \eta$. An analogous plot for $c_{2}$ is shown in figure 9 that depicts comparable features. In general, the magnitude of this parameter with respect to its counterparts is smaller.

For a dispersion of Janus drops with identical orientations it is obvious that $\left\langle S_{i j}^{p}\right\rangle=n_{d} S_{i j}^{p}$, where $n_{d}$ is the number density of the drops. Thus, the constitutive equation for a dilute Janus emulsion becomes:

$$
\begin{aligned}
& \left\langle\Sigma_{i j}\right\rangle=-\langle p\rangle \delta_{i j}+2 \eta_{0}\left(1+\frac{1+\frac{5}{2} \eta}{1+\eta} \Phi\right) E_{i j}^{\infty} \\
& +\frac{1}{2} \eta_{0} c_{1} \Phi\left[\left(d_{i} E_{j k}^{\infty}+d_{j} E_{i k}^{\infty}\right) d_{k}-2 d_{i} d_{j} d_{k} d_{l} E_{l k}^{\infty}\right] \\
& +\frac{1}{2} \eta_{0} c_{2} \Phi\left(\epsilon_{i k l} d_{j}+\epsilon_{j k l} d_{i}\right) d_{l}\left(\Omega_{k}^{\infty}-\omega_{k}\right),
\end{aligned}
$$



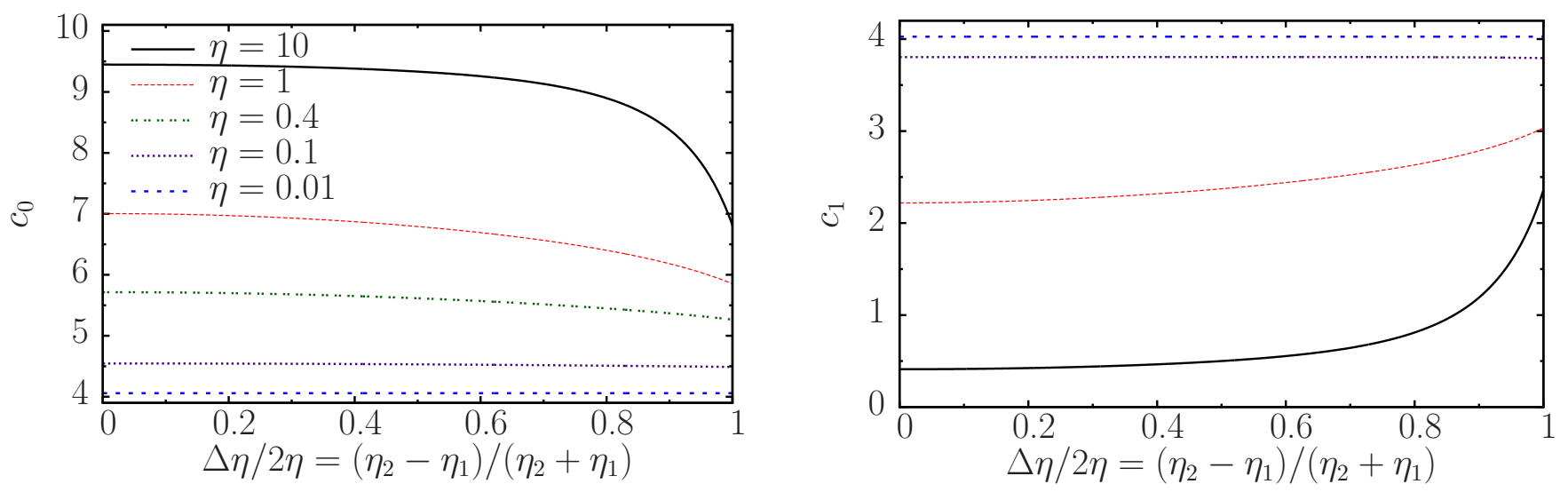

Figure 8: (Colors online) Variation of the stresslet functions with the viscosity contrast $\Delta \eta / 2 \eta$ for selected values of the mean drop viscosity.

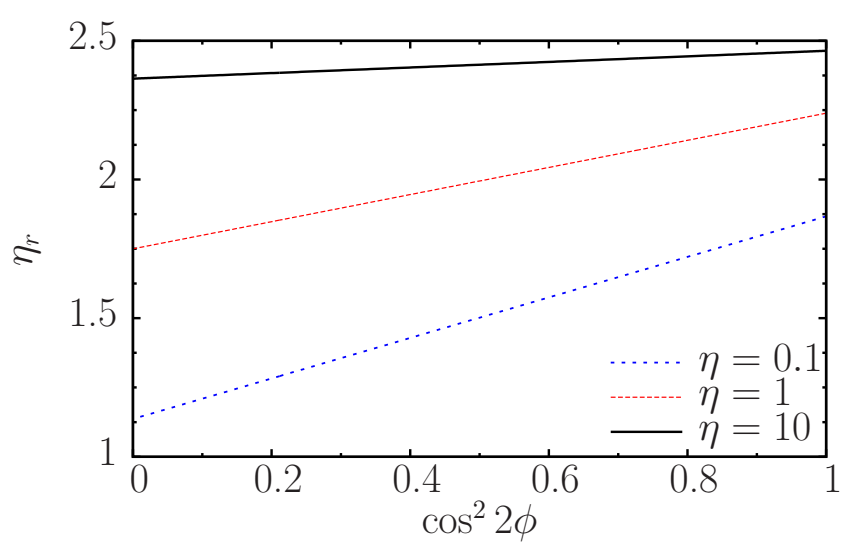

Figure 10: The reduced effective viscosity $\eta_{r}$ of a dilute Janus emulsion as a function of the drop mean viscosity and orientation. The minimum values of $\eta_{r}$ (at $\left.\cos ^{2} 2 \phi=0\right)$ correspond to Taylor's result for single-phase drops. The axis of symmetry of the dispersed Janus drops $\boldsymbol{d}$ lie in the plane of shear.

where $\Phi$ is the volume fraction occupied by the Janus drops. The orientation-independent contribution to the bulk stress (second term) is the effective viscosity of a dilute dispersion of single-phase drops (Taylor, 1932) with an equivalent viscosity $\eta=\left(\eta_{1}+\eta_{2}\right) / 2$. It is interesting to note that this term corresponds to the minimum rate of viscous dissipation for a dilute Janus emulsion. Figure 10 presents the variation of the reduced effective viscosity $\eta_{r}=\left(\left\langle\tilde{\Sigma}_{12}\right\rangle-G \tilde{\eta}_{0}\right) / G \tilde{\eta}_{0} \Phi$ of the dispersion with the drop viscosity $\eta$ and orientation when $\boldsymbol{d}$ is in the plane of shear. From this figure it is evident that the effective viscosity of a Janus emulsion is intermediate to that of a dispersion of low viscosity drops and hard spheres at infinite dilution conditions. The dependence of the orientation of the Janus drops on the effective viscosity becomes unimportant as $\eta \rightarrow \infty$, in agreement with the expecta- tions. The somewhat surprising result is that there is a stronger dependence of the effective viscosity on the drop orientation $\boldsymbol{d}$ for Janus drops of low viscosity $(\eta \ll 1)$. In contrast to a single-phase drop, a Janus drop that rotates produces a disturbance-field that originates from the hindrance of the internal interface to the flow that is present even in the low viscosity limit.

It is pertinent to compare the obtained effective viscosity against that of double emulsions, a dispersion of concentric drops, in an attempt to obtain a broader picture of the rheology of compound multiphase drops. On account of the concentric configuration of double emulsions the effective viscosity is, in general, more sensitive to the separation of the interfaces than the viscous properties of the constituent fluids (Stone and Leal, 1990). For example, the viscosity of a dispersion of hard spheres is recovered for small interfacial separations of the concentric drops with a lesser dependence on the viscosity of the internal fluids (for moderate values). Only when the inner drop size is negligible compared to the outer one, Taylor's viscosity is recovered, as pointed out by Stone and Leal (1990). Therefore the consolidation of these results with the present ones indicate that the lower and upper bounds to the viscosity of a dispersion of compound multiphase drops, in general, correspond to those of single-phase drops and hard spheres, respectively.

The remarkable difference of the rheological properties of Janus emulsions to double emulsions is the appearance of non-Newtonian effects associated to the anisotropic hydrodynamics of Janus drops. Whereas, double emulsions exhibit Newtonian behavior (isotropic bulk stress) in the limit of zero interfacial distortions (Stone and Leal, 1990). The difference in the normal stresses $N_{1}=\left\langle\Sigma_{11}\right\rangle-\left\langle\Sigma_{22}\right\rangle$ and $N_{2}=\left\langle\Sigma_{22}\right\rangle-\left\langle\Sigma_{33}\right\rangle$ of a dilute Janus emulsion in a lin- 
ear shear flow are readily determined from the bulk stress:

$$
\begin{aligned}
& N_{1}=\eta_{0} c_{1} \Phi\left(d_{2}^{2}-d_{1}^{2}\right) d_{1} d_{2} E_{12}^{\infty}, \\
& N_{2}=\eta_{0} c_{1} \Phi\left[\frac{1}{2}+\left(d_{3}^{2}-d_{2}^{2}\right)\right] d_{1} d_{2} E_{12}^{\infty} .
\end{aligned}
$$

As one may expect, from the intrinsic analogy of spheroids of revolution, there is no direct contribution to the difference in normal stresses from the rotational motions of $f_{590}$ the Janus drops; the influence of rotation to the normal stresses is as an indirect contribution via changes in orientation $\boldsymbol{d}$. The difference in the first normal stresses $N_{1}$ vanishes identically when the axis of symmetry of the Janus drop is either parallel to the direction of the undisturbed ${ }_{595}$ flow or velocity-gradient (i.e. $d_{1,2}=1$ ), and when the axis of symmetry is parallel to any of the principal axes of the rate-of-strain field $\left(d_{1}= \pm d_{2}\right)$. This is quite the expected result for any spheroidal particle on account of the rotational symmetry about its axis of symmetry. In fact, dilute $e_{60}$ dispersions of other related bodies of revolution of spherical shape but with anisotropic hydrodynamic properties (e.g. 'slip-stick' spheres) also exhibit the analogous behavior of $N_{1}$ in a linear shear flow (Ramachandran and Khair, 2009). For other arrangements of $\boldsymbol{d}$, the Janus drop expe-605 riences an uneven distribution of the normal stresses over its outer surface. The transition of $N_{1}$ from compression to tensile normal stresses is consistent to that of an oblate spheroidal particle, in agreement with the expectations for a body that exhibits hydrodynamic properties analogous 610 to that of an oblate spheroid (Section 4). The second difference in the normal stresses $N_{2}$ vanishes wherever $\boldsymbol{d}$ is aligned with any of the principal axes of the undisturbed flow (velocity and velocity-gradient) or rate-of-strain field $d_{3}=1$ the dispersion behaves as an emulsion with Newtonian properties $\left(N_{1,2}=0\right)$ because in this case the Janus drop behaves as a single-phase drop.

It is relevant to point out that differences in the nor-

mal stresses can also arise in dispersions of single-phase ${ }_{620}$ drops; however, within the limit of small deflections these are of the order of the capillary number $C a \ll 1$ (Schowalter et al., 1968). From this result, it ensues that the effects of the deflection of the interfaces may not be easily

570 differentiated in experimental settings from those tied to $_{625}$ the anisotropic properties of the Janus drops as the capillary number is increased. The obtained results indicate that the anisotropic properties of the Janus drop provide the leading term for the differences in the normal stresses,

575 which establishes a reasonable justification for the neglect ${ }_{630}$ interface distortions in this study. It is also important to point out that Torza and Mason (1970) did not observed qualitative changes on the dynamical behavior of a near Janus drops with increasing shear rate despite the notable

580 deformation to the interfaces. The analysis of shape dis-635 tortions is expected to be more meaningful when there are hydrodynamic interactions with other drops or walls and in time-dependent shear flows, as it has been for sim- ple fluid drops (Frankel and Acrivos, 1970; Barthès-Biesel and Acrivos, 1973; Magnaudet et al., 2003).

\section{Conclusions}

The dynamics and rheology of Janus drops in a linear shear flow of a Newtonian fluid is studied in the limit of low-Reynolds-number flows. Under 'freely' suspended conditions the Janus drops undergo Jeffery-like orbits analogous to that of an oblate spheroidal particle with an effective aspect ratio determined by the viscosities of the three fluid phases. Surprisingly, low viscous Janus drops formed by distinct fluids tend to exhibit disk-like Jeffery orbits, and as one may anticipate, high viscous drops behave as solid spheres (isotropic).

When a Janus drop is 'trapped' at a fixed location by the action of an external force it can exhibit a preferential orientation depending on the viscous properties of the fluids, velocity, and velocity-gradient of the undisturbed flow. This property may be particularly useful to estimate the local velocity to velocity-gradient in an unknown ambient flow, and to obtain a dispersion of Janus drops with a uniform orientation distribution. The latter is of practical importance to the study of the changes in the rheology of Janus emulsions with respect to the orientation of the drops.

This investigation shows that an external force parallel to the streamlines of the undisturbed shear flow can induce a cross-streaming velocity on the Janus drop. This has interesting implications in a dispersion of Janus drops subject to a pressure-driven flow. When the dispersion is forced to flow in a tube by a pressure head (confined Poiseuille flow), the drops are expected to migrate away from the center to the walls of the tube by means of an external force (e.g. gravity) parallel to the axis of the tube. Near the center of the tube the shear-to-force ratio is small such that the drops undergo cross-flow migration until a zone of higher shear is reached, in which the drops rotation is fast enough to prevent further migration. It is likely that the hydrodynamic interactions with the wall will also affect the location of drops relative to the axis of the tube in this case. This establishes a pure hydrodynamic approach to the separation of multiphase emulsions with potential applications in environmental remediation processes. Additionally, the tendency of Janus drops to approach the boundaries of a tube in a pressure-driven flow can have medical applications. For example, Janus drops can be made selective for the capture of hazardous lipids that accumulate near the walls of blood vessels as a method to reduce cholesterol levels, which is one of the related factors that lead to cardiovascular diseases in the United States (Lichtenstein et al., 2006).

The rheological behavior of Janus drops has been found to share properties with dispersions of single-phase drops and spheroidal particles. Interestingly, the expression of the effective viscosity is analogous to that derived by Taylor (1932) for a dispersion of single-phase drops, but with 
a drop viscosity equivalent to the mean of the viscosithe pair of fluids that comprise the Janus drop, $\eta=\left(\eta_{1}+\eta_{2}\right) / 2$. This corresponds to the minimum rate of viscous dissipation for this system, which occurs when the axis of symmetry of the Janus drop is either parallel ${ }_{695}$ to the vorticity axis or any of the principal axes of the te-of-strain field. The effect of the viscosity contrast of the internal fluids is in general small such that it can be safely neglected in most experimental settings that have been reported in the scientific literature. In comparison to a double emulsion (concentric drops), a dispersion of Janus 650 drops displays non-Newtonian behavior that derives from the anisotropic properties imparted by the internal fluidfluid interface. Although this study has only considered the rheological properties under 'freely' suspended conditions, it is not difficult to apply the results to conditions in 655 which the orientation of the Janus drops is controlled by external means (e.g. magnetic field). Additionally, there ${ }_{710}$ would be an anti-symmetric contribution to the bulk stress intrinsically related to the applied torque; the fundamental relation can be found in the well-known book of Kim and Karrila (2005), such that in principle, one can still de- ${ }_{715}$ termine the effective viscosity by accounting for the contribution of the torque to the bulk stress of the dispersion. Nevertheless, it would be interesting to compare the theory presented here to rheological measurements from experi- ${ }_{720}$ ments to advance the understanding of the rich dynamics of multiphase emulsions.

The results of this investigation can also be useful to estimate and understand the dynamics and rheology of other ${ }_{725}$ partly engulfed multiphase drops. This expectation is partially supported by the experimental observations carried out by Torza and Mason (1970), where it is demonstrated that the dynamics of such drops is similar to that of a $\mathrm{a}_{30}$ spheroid of revolution. It follows from the findings of this study that the effective viscosity of a dispersion of partly engulfed compound drops may be comparable to Taylor's result under certain circumstances. If the fluids that con-735 stitute the partly engulfed drop occupy similar volumes, as for the particular case of a Janus drop, it is likely that Taylor's expression can be used to predict the effective viscosity. For other volume ratios the extrema of a single-740 phase drop and a solid sphere are expected with a lesser influence of the internal viscosities for short separations of the fluid-fluid interfaces.

\section{Acknowledgements}

685 We are very grateful to Dr. Sergey Shklyaev for bringing750 to our attention the problem of preferential orientation of the Janus drop. This publication is based on work supported by Award No. RUP1-7078-PE12 (joint grant with Ural Branch of the Russian Academy of Sciences) of the $\mathrm{e}^{755}$ ${ }_{690}$ U.S. Civilian \& Development Foundation (CRDF Global) and by the National Science Foundation under Cooperative Agreement No. OISE-9531011. This work was partly supported by the National Science Foundation; CAREER Award (CBET-1055284).

\section{References}

Barthès-Biesel, D., Acrivos, A., 1973. Deformation and burst of a liquid droplet freely suspended in a linear shear field. J. Fluid Mech. 61, 1-22.

Batchelor, G. K., 1970. The stress system in a suspension of forcefree particles. J. Fluid Mech. 41, 545-570.

Bazhlekov, I., Shopov, P., Zapryanov, Z., 1995. Unsteady motion of a type-A compound multiphase drop at moderate Reynolds numbers. J. Coll. Int. Sci. 169, 1-12.

Brenner, H., 1963. The Stokes resistance of an arbitrary particle. Chem. Engng. Sci. 18, 1-25.

Brenner, H., 1974. Rheology of a dilute suspension of axisymmetric Brownian particles. Int. J. Mult. Flow 1, 195-341.

Bretherton, F., 1962. The motion of rigid particles in a shear flow at low Reynolds number. J. Fluid Mech. 14, 284-304.

Bringer, M., Gerdts, C., Song, H., Tice, J., Ismagilov, R., 2004. Microfluidic systems for chemical kinetics that rely on chaotic mixing in droplets. Phil. Trans. Roy. Soc. Lon. A: Math., Phys. Engng. Sci. 362 (1818), 1087-1104.

Chan, P., Leal, L., 1979. The motion of a deformable drop in a second-order fluid. J. Fluid Mech. 92, 131-170.

Chervenivanova, E., Zapryanov, Z., 1989. On the deformation of compound multiphase drops at low Reynolds numbers. PhysicoChem. Hydrodyn. 11, 243-259.

Choi, C.-H., Hwang, S., Jeong, J.-M., Kang, S.-M., Kim, J., Lee, C.S., 2012. Microfluidic synthesis of anisotropic particles from Janus drop by in situ photopolymerization. Biomed. Engng. Letters 2, 95-99.

Davis, A., Brenner, H., 1981. Emulsions containing a third solid internal phase. J. Engng. Mech. Div. ASCE 107, 609-621.

Deen, W. M., 1998. Analysis of Transport Phenomena. Oxford University Press.

Dendukuri, D., Doyle, P., 2009. The synthesis and assembly of polymeric microparticles using microfluidics. Adv. Mater. 21, 40714086.

Derkach, S., 2009. Rheology of emulsions. Adv. Coll. Int. Sci. 151, $1-23$.

Díaz-Maldonado, M., Córdova-Figueroa, U. M., 2015. On the anisotropic response of a Janus drop in a shearing viscous fluid. J. Fluid Mech. 770, R2.

URL http://dx.doi.org/10.1017/jfm.2015.148

Dorrepaal, J., 1978. The Stokes resistance of a spherical cap to translational and rotational motions in a linear shear flow. J. Fluid Mech. 84, 265-279.

Frankel, N., Acrivos, A., 1970. The constitutive equation for a dilute emulsion. J. Fluid Mech. 44, 65-78.

Guzowski, J., Korczyk, P. M., Jakiela, S., Garstecki, P., 2012. The structure and stability of multiple micro-droplets. Soft Matter 8, 7269-7278.

Happel, J., Brenner, H., 1965. Low Reynolds Number Hydrodynamics: With special applications to particulate media. Prentice-Hall.

Hinch, E., Leal, L., 1973. Time-dependent shear flows of a suspension of particles with weak Brownian rotations. J. Fluid Mech. 57, 753767 .

Hoskins, M., Kunz, R., Bistline, J., Dong, C., 2009. Coupled flowstructure-biochemistry simulations of dynamic systems of blood cells using an adaptive surface tracking method. J. Fluids and Struct. 25, 936-953.

Hua, H., Shin, J., Kim, J., 2014. Dynamics of a compound droplet in shear flow. Int. J. Heat Fluid Flow 50, 63-71.

Jeffery, G., 1922. The motion of ellipsoidal particles immersed in a viscous fluid. Proc. R. Soc. Lond., Ser. A 102, 161-179.

Jeong, J., Gross, A., Wei, W.-S., Tu, F., Lee, D., Collings, P., Yodh, A., 2015. Liquid crystal Janus emulsion droplets: preparation, tumbling, and swimming. Soft Matter 11, 6747-6754. 

single-cell analysis. Ang. Chem. Int. Ed. 51, 12176-12192.

Johnson, R., Sadhal, S., 1985. Fluid mechanics of compound multiphase drops and bubbles. Annu. Rev. Fluid Mech. 17, 289-320.

Kan, H.-C., Udaykumar, H., Shyy, W., Tran-Son-Tay, R., 1998. Hy-835 drodynamics of a compound drop with application to leukocyte modeling. Phys. Fluids 10, 760-774.

Kim, S., Karrila, S., 2005. Microhydrodynamics Principles and Selected Applications. Dover.

Larson, R., 1999. The Structure and Rheology of Complex Fluids.840 Oxford University Press.

Leal, L., 1980. Particle motions in a viscous fluid. Annu. Rev. Fluid Mech. 12, 435-476.

Lee, Y., Kim, C., Jang, W., Choi, H., Jhon, M., 2001. Synthesis and electrorheological characteristics of microencapsulated polyaniline 845 particles with melamine-formaldehyde resins. Polymer 42, 82778283.

Li, N., 1971. Separation of hydrocarbons by liquid membrane permeation. Ind. Engng. Chem. Proc. Des. Dev. 10, 215-221.

Lichtenstein, A., Appel, L., Brands, M., Carnethon, M., Daniels, S., 850 Franch, H., Franklin, B., Kris-Etherton, P., Harris, W., Howard, B., Karanja, N., Lefevre, M., Rudel, L., Sacks, F., Van Horn, L., Winston, M., Wylie-Rosett, J., 2006. Diet and lifestyle recommendations revision 2006: A scientific statement from the American heart association nutrition committee. Circulation 114, 82-96. 855

Magnaudet, J., Takagi, S., Legendre, D., 2003. Drag, deformation and lateral migration of a buoyant drop moving near a wall. J. Fluid Mech. 476, 115-157.

Morton, D., Subramanian, R., Balasubramaniam, R., 1990. The migration of a compound drop due to thermocapillarity. Phys. Fluids860 A 2, 2119-2133.

Nir, A., Acrivos, A., 1973. On the creeping motion of two arbitrarysized touching spheres in a linear shear field. J. Fluid Mech. 59, 209-223.

Nisisako, T., Torii, T., Takahashi, T., Takizawa, Y., 2006. Synthesis of monodisperse bicolored Janus particles with electrical anisotropy using a microfluidic co-flow system. Adv. Mater. 18, $1152-1156$

Olabi, A., Grunwald, A., 2007. Design and application of magnetorheological fluid. Mater. Design 28, 2658-2664.

$\mathrm{Qu}, \mathrm{X}$., Wang, Y., 2012. Dynamics of concentric and eccentric compound droplets suspended in extensional flows. Phys. Fluids 24, 123302 .

Ramachandran, A., Khair, A., 2009. The dynamics and rheology of a dilute suspension of hydrodynamically Janus spheres in a linear flow. J. Fluid Mech. 633, 233-269.

Rosenfeld, L., Lavrenteva, O., Nir, A., 2009. On the thermocapillary motion of partially engulfed compound drops. J. Fluid Mech. 626, 263-289.

Russel, W., Saville, D., Schowalter, W., 1989. Colloidal Dispersions. Cambridge University Press.

Sadhal, S., Oguz, H., 1985. Stokes flow past compound multiphase drops: the case of completely engulfed drops/bubbles. J. Fluid Mech. 160, 511-529.

Saffman, P., 1956. On the motion of small spheroidal particles in a viscous liquid. J. Fluid Mech. 1, 540-553.

Schowalter, W., Chaffey, C., Brenner, H., 1968. Rheological behavior of a dilute emulsion. J. Coll. Int. Sci. 26, 152-160.

Shardt, O., Derksen, J., Mitra, S., 2014. Simulations of Janus droplets at equilibrium and in shear. Phys. Fluids 26, 012104.

Shintaku, H., Kuwabara, T., Kawano, S., Suzuki, T., Kanno, I., Kotera, H., 2007. Micro cell encapsulation and its hydrogel-beads production using microfluidic device. Microsys. Tech. 13, 951-958.

Shklyaev, S., 2015. Janus droplet as a catalytic micromotor. Europhys. Letters 110, 54002.

Shklyaev, S., Ivantsov, A., Díaz-Maldonado, M., Córdova-Figueroa, U. M., 2013. Dynamics of a Janus drop in an external flow. Phys. Fluids 25, 082105.

URL http://dx.doi.org/10.1063/1.4817541

Smith, K., Ottino, J., Olvera de la Cruz, M., 2004. Encapsulated 830
Stone, H., Leal, L., 1990. Breakup of concentric double emulsion droplets in linear flows. J. Fluid Mech. 211, 123-156.

Summers, M., Reid, J., McGloin, D., 2006. Optical guiding of aerosol droplets. Opt. Express 14, 6373-6380.

Taylor, G., 1932. The viscosity of a fluid containing small drops of another fluid. Proc. R. Soc. A 138, 41-48.

Tian, R., Zheng, Y., Liang, X., Zhou, Z., Fu, X., Lv, W., 2011. The preparation of microcapsules used for self-healing composites. Advanced Materials Research 233-235, 2319-2322.

Torza, S., Mason, S., 1970. Three-phase interactions in shear and electrical fields. J. Coll. Int. Sci. 33, 67-83.

Tsemakh, D., Lavrenteva, O., Nir, A., 2004. On the locomotion of a drop, induced by the internal secretion of surfactant. Int. J. Multiphase Flow 30, 1337-1367.

Vehring, R., 2008. Pharmaceutical particle engineering via spray drying. Pharm. Res. 25, 999-1022.

Vuong, S., Sadhal, S., 1989. Growth and translation of a liquidvapour compound drop in a second liquid. Part 1. Fluid mechanics. J. Fluid Mech. 209, 617-637.

Wang, J.-T., Wang, J., Han, J.-J., 2011. Fabrication of advanced particles and particle-based materials assisted by droplet-based microfluidics. Small 7, 1728-1754.

Zarzar, L., Sresht, V., Sletten, E., Kalow, J., Blankschtein, D., Swager, T., 2015. Dynamically reconfigurable complex emulsions via tunable interfacial tensions. Nature 518, 520-524.

Zhou, C., Yue, P., Feng, J., 2006. Formation of simple and compound drops in microfluidic devices. Phys. Fluids 18, 092105.

Zhou, C., Yue, P., Feng, J., 2008. Deformation of a compound drop through a contraction in a pressure-driven pipe flow. Int. J. Mult. Flow 34, 102-109. 